\title{
Magnetic and electric contributions to the energy loss in a dynamical QCD medium
}

\author{
Magdalena Djordjevic* \\ Institute of Physics Belgrade, University of Belgrade, Serbia
}

(Dated: November 26, 2018)

\begin{abstract}
The computation of radiative energy loss in a finite size QCD medium with dynamical constituents is a key ingredient for obtaining reliable predictions for jet quenching in ultra-relativistic heavy ion collisions. It was previously shown that energy loss in dynamical QCD medium is significantly higher compared to static QCD medium. To understand this difference, we here analyze magnetic and electric contributions to energy loss in dynamical QCD medium. We find that the significantly higher energy loss in the dynamical case is entirely due to appearance of magnetic contribution in the dynamical medium. While for asymptotically high energies, the energy loss in static and dynamical medium approach the same value, we find that the physical origin of the energy loss in these two cases is different.
\end{abstract}

PACS numbers: 25.75.-q, 25.75.Nq, 12.38.Mh, 12.38.Qk

\section{INTRODUCTION}

Jet suppression [1] is considered to be a powerful tool to study the properties of a QCD medium created in ultrarelativistic heavy ion collisions [2 -4] . The suppression results from the energy loss of high energy partons moving through the plasma [5 8 ]. Therefore, reliable computations of jet energy loss mechanisms are essential for the reliable predictions of jet suppression. In 9, 10], we developed a theoretical formalism for the calculation of the first order in opacity light and heavy quark radiative energy loss in dynamical QCD medium (see also a viewpoint [1]]). That study, which incorporates dynamical effects in realistic finite size QCD medium, enables us to provide the most reliable computations of the energy loss in QGP so far. This work has shown that the energy loss in dynamical medium is significantly larger (50-70\%), compared to the energy loss in static QCD medium.

The goal of this paper is to determine what is the origin of the observed significant energy loss increase in the case of dynamical QCD medium. To that end, we here analyze magnetic and electric contributions to the energy loss in the dynamical QCD medium, under conditions relevant at RHIC and LHC experiments. We furthermore analyze what happens for asymptotically large energies, where it was previously shown that the energy loss in static and dynamical QCD medium takes the same value [9, 10].

\section{CONTRIBUTIONS TO RADIATIVE ENERGY LOSS IN A DYNAMICAL QCD MEDIUM}

We here study the importance of electric and magnetic contributions to the medium induced radiative energy loss in a finite size dynamical QCD medium. In [9, 10] it was shown that the radiative energy loss in a finite size dynamical QCD medium is given by:

$$
\frac{\Delta E^{\mathrm{dyn}}}{E}=2 \frac{C_{R} \alpha_{s}}{\pi} \frac{L}{\lambda_{\mathrm{dyn}}} \int d x \frac{d^{2} k}{\pi} \frac{d^{2} q}{\pi} v(\boldsymbol{q})\left(1-\frac{\sin \frac{(\boldsymbol{k}+\boldsymbol{q})^{2}+\chi}{x E^{+}} L}{\frac{(\boldsymbol{k}+\boldsymbol{q})^{2}+\chi}{x E^{+}} L}\right) \frac{(\boldsymbol{k}+\boldsymbol{q})}{(\boldsymbol{k}+\boldsymbol{q})^{2}+\chi}\left(\frac{(\boldsymbol{k}+\boldsymbol{q})}{(\boldsymbol{k}+\boldsymbol{q})^{2}+\chi}-\frac{\boldsymbol{k}}{\boldsymbol{k}^{2}+\chi}\right),
$$

Here $L$ is the length of the finite size dynamical QCD medium and $E$ is the jet energy. $k$ is transverse momentum of radiated gluon, while $\boldsymbol{q}$ is transverse momentum of the exchanged (virtual) gluon. $\alpha_{s}=\frac{g^{2}}{4 \pi}$ is coupling constant and $C_{R}=\frac{4}{3} \cdot v(\boldsymbol{q})$ is the effective crossection in dynamical QCD medium and $\lambda_{\text {dyn }}^{-1} \equiv C_{2}(G) \alpha_{s} T=3 \alpha_{s} T\left(C_{2}(G)=3\right)$ is defined as "dynamical mean free path" (see also [12]). $\chi \equiv M^{2} x^{2}+m_{g}^{2}$, where $x$ is the longitudinal momentum fraction of the heavy quark carried away by the emitted gluon, $M$ is the mass of the heavy quark, $m_{g}=\mu_{E} / \sqrt{2}$ is the effective mass for gluons with hard momenta $k \gtrsim T$ and $\mu_{E}$ is the Debye mass. We assume constant coupling $g$.

The effective crossection $v(\boldsymbol{q})$ can be written in the following form

$$
v(\boldsymbol{q})=v_{L}(\boldsymbol{q})+v_{T}(\boldsymbol{q}),
$$

\footnotetext{
*Correspond to magda@ipb.ac.rs
} 
where $v_{L}(\boldsymbol{q})\left(v_{T}(\boldsymbol{q})\right)$ is longitudinal (transverse) contribution to the effective crossection, given by [9, 13]

$$
\begin{aligned}
& v_{L}(\boldsymbol{q})=\frac{1}{\boldsymbol{q}^{2}+\operatorname{Re} \Pi_{L}(\infty)}-\frac{1}{\boldsymbol{q}^{2}+\operatorname{Re} \Pi_{L}(0)} \\
& v_{T}(\boldsymbol{q})=\frac{1}{\boldsymbol{q}^{2}+\operatorname{Re} \Pi_{T}(0)}-\frac{1}{\boldsymbol{q}^{2}+\operatorname{Re} \Pi_{T, L}(\infty)} .
\end{aligned}
$$

$\Pi_{L}$ and $\Pi_{T}$ are HTL gluon self energies, given by 14

$$
\Pi_{L}(l)=\mu_{E}^{2}\left[1-y^{2}-\frac{y\left(1-y^{2}\right)}{2} \ln \left(\frac{y+1}{y-1}\right)\right], \quad \Pi_{T}(l)=\mu_{E}^{2}\left[\frac{y^{2}}{2}+\frac{y\left(1-y^{2}\right)}{4} \ln \left(\frac{y+1}{y-1}\right)\right],
$$

where $y \equiv l_{0} /|\overrightarrow{\mathbf{I}}|\left(l\right.$ is the momentum of the gluon) and $\mu_{E}$ is Debye screening mass.

By using the following properties of the HTL self-energy of the gluon [15]

$$
\begin{aligned}
& \operatorname{Re} \Pi_{T, L}(\infty)=\mu_{\infty}^{2} \\
& \operatorname{Re} \Pi_{T}(0)=0 \\
& \operatorname{Re} \Pi_{L}(0)=\mu_{E}^{2},
\end{aligned}
$$

where $\mu_{\infty} \equiv \mu_{E} / \sqrt{3}$ is the gluon thermal mass, we obtain

$$
\begin{aligned}
& v_{L}(\boldsymbol{q})=\frac{1}{\left(\boldsymbol{q}^{2}+\mu_{\infty}^{2}\right)}-\frac{1}{\left(\boldsymbol{q}^{2}+\mu_{E}^{2}\right)}=\frac{\mu_{E}^{2}-\mu_{\infty}^{2}}{\left(\boldsymbol{q}^{2}+\mu_{\infty}^{2}\right)\left(\boldsymbol{q}^{2}+\mu_{E}^{2}\right)} \\
& v_{T}(\boldsymbol{q})=\frac{1}{\boldsymbol{q}^{2}}-\frac{1}{\left(\boldsymbol{q}^{2}+\mu_{\infty}^{2}\right)}=\frac{\mu_{\infty}^{2}}{\boldsymbol{q}^{2}\left(\boldsymbol{q}^{2}+\mu_{\infty}^{2}\right)} .
\end{aligned}
$$

After replacing the effective crossection in Eq. (2.1), with expressions from Eqs. (2.6), we obtain magnetic (transverse) and electric (longitudinal) contributions to the energy loss:

$$
\begin{aligned}
\frac{\Delta E_{\mathrm{M}, \mathrm{E}}^{d y n}}{E}=\frac{C_{R} \alpha_{s}}{\pi} \frac{L}{\lambda_{\mathrm{dyn}}} \int d x \frac{d^{2} k}{\pi} \frac{d^{2} q}{\pi} v_{T, L}(\boldsymbol{q})\left(1-\frac{\sin \frac{(\boldsymbol{k}+\boldsymbol{q})^{2}+\chi}{x E^{+}} L}{\frac{(\boldsymbol{k}+\boldsymbol{q})^{2}+\chi}{x E^{+}} L}\right) \\
\quad \times 2 \frac{(\boldsymbol{k}+\boldsymbol{q})}{(\boldsymbol{k}+\boldsymbol{q})^{2}+\chi}\left(\frac{(\boldsymbol{k}+\boldsymbol{q})}{(\boldsymbol{k}+\boldsymbol{q})^{2}+\chi}-\frac{\boldsymbol{k}}{\boldsymbol{k}^{2}+\chi}\right),
\end{aligned}
$$

In the above equation $v_{T}(\boldsymbol{q})$ under the integral corresponds to the magnetic contribution $\left(\Delta E_{\mathrm{M}}^{d y n}\right)$, while $v_{L}(\boldsymbol{q})$ corresponds to the electric contribution $\left(\Delta E_{\mathrm{E}}^{d y n}\right)$. From Eq. (2.6), we see that Debye screening $\left(\mu_{E}\right)$ renders the longitudinal gluon exchange $\left(v_{L}(\boldsymbol{q})\right)$ infrared finite, leading to a finite electric contribution to the energy loss Eq. (2.7). The transverse gluon exchange $\left(v_{T}(\boldsymbol{q})\right.$ in Eq. (2.6) $)$ causes a well-known logarithmic singularity [16], due to the absence of a magnetic screening. However, one should note that the magnetic contribution $\left(\Delta E_{\mathrm{M}}^{d y n}\right)$ has to be finite as well, since in [9, 10, 12] it was shown that the total energy loss in dynamical QCD medium is finite. Therefore, we can use Eqs. (2.6) and (2.7) to numerically study electric and magnetic contributions to dynamical energy loss, which will be done in Section IV]

\section{ASYMPTOTIC LIMIT OF HIGH ENERGIES}

We here analyze magnetic and electric contributions to the dynamical energy loss in the limit of high energies. The limit is obtained from Eq. (2.7) when $E^{+} \approx 2 E \rightarrow \infty$. In such a limit finite mass effects are negligible. Additionally, $k_{\max }=2 E \sqrt{x(1-x)} \rightarrow \infty$ as well, which enables us to introduce a substitution $\boldsymbol{k}^{\prime} \equiv \boldsymbol{k}+\boldsymbol{q}$ in Eq. (2.7). With these simplifications, Eq. (2.7) becomes

$$
\frac{\Delta E_{\mathrm{M}, \mathrm{E}}^{\mathrm{dyn}}}{E}=\frac{C_{R} \alpha_{s}}{\pi} \frac{L}{\lambda_{\mathrm{dyn}}} \int d x \frac{d^{2} k^{\prime}}{\pi} \frac{d^{2} q}{\pi} v_{T, L}(\boldsymbol{q}) \frac{2 \boldsymbol{q} \cdot\left(\boldsymbol{q}-\boldsymbol{k}^{\prime}\right)}{\boldsymbol{k}^{\prime 2}\left(\boldsymbol{k}^{\prime}-\boldsymbol{q}\right)^{2}}\left(1-\frac{\sin \left(\frac{\boldsymbol{k}^{\prime 2} L}{2 x E}\right)}{\frac{\boldsymbol{k}^{\prime 2} L}{x E^{+}}}\right)
$$

where $v_{T, L}(\boldsymbol{q})$ is given by Eq. (2.6). 
After performing the angular integration, taking the derivative over distance $L$ of the above expression and performing the integral over $\boldsymbol{k}^{\prime 2}$, we obtain

$$
\frac{1}{E} \frac{d E_{\mathrm{M}, \mathrm{E}}^{\mathrm{dyn}}}{d L}=2 \frac{C_{R} \alpha_{s}}{\pi} \frac{1}{\lambda_{\mathrm{dyn}}} \int_{0}^{q_{\text {max }}^{2}} d \boldsymbol{q}^{2} \int_{0}^{1} d x v_{T, L}(\boldsymbol{q})\left(\gamma-\mathrm{Ci}\left(\frac{L \boldsymbol{q}^{2}}{2 E x}\right)+\ln \left(\frac{L \boldsymbol{q}^{2}}{2 E x}\right)\right),
$$

where $\gamma \approx 0.577216$ is Euler's constant and $\mathrm{Ci}(y)$ is the cosine integral function.

Finally after performing the integration over $x$, we obtain (in the limit when $E \rightarrow \infty$ )

$$
\frac{1}{E} \frac{d E_{\mathrm{M}, \mathrm{E}}^{\mathrm{dyn}}}{d L}=\frac{C_{R} \alpha_{s}}{2 E} \frac{L}{\lambda_{\mathrm{dyn}}} \int_{0}^{q_{\max }^{2}} d \boldsymbol{q}^{2} \boldsymbol{q}^{2} v_{T, L}(\boldsymbol{q})
$$

Therefore, for the asymptotically large jet energies, the Eq. (3.3) reduces to

$$
\begin{aligned}
& \frac{1}{E} \frac{d E_{\mathrm{M}}^{\mathrm{dyn}}}{d L}=\frac{C_{R} \alpha_{s}}{2 E} \frac{L}{\lambda_{\mathrm{dyn}}} \mu_{\infty}^{2} \ln \left(\frac{4 E T}{\mu_{\infty}^{2}}\right), \\
& \frac{1}{E} \frac{d E_{\mathrm{E}}^{\mathrm{dyn}}}{d L}=\frac{C_{R} \alpha_{s}}{2 E} \frac{L}{\lambda_{\mathrm{dyn}}}\left[\mu_{E}^{2} \ln \left(\frac{4 E T}{\mu_{E}^{2}}\right)-\mu_{\infty}^{2} \ln \left(\frac{4 E T}{\mu_{\infty}^{2}}\right)\right],
\end{aligned}
$$

where we used $q_{\max }=\sqrt{4 E T}[17$ ]. Finally, fractional energy loss then becomes

$$
\begin{gathered}
\frac{\Delta E_{\mathrm{M}}^{\mathrm{dyn}}}{E}=\frac{C_{R} \alpha_{s}}{4 E} \frac{L^{2} \mu_{\infty}^{2}}{\lambda_{\mathrm{dyn}}} \ln \left(\frac{4 E T}{\mu_{E}^{2}}\right), \\
\frac{\Delta E_{\mathrm{E}}^{\mathrm{dyn}}}{E} \approx \frac{\Delta E_{\mathrm{M}}^{\mathrm{dyn}}}{E} \frac{\mu_{E}^{2}-\mu_{\infty}^{2}}{\mu_{\infty}^{2}} .
\end{gathered}
$$

From the above two equations, it directly follows that, in the asymptotic limit, the ratio of magnetic and electric contributions to the dynamical QCD medium is equal to $\frac{1}{2}$ (i.e. $\frac{\mu_{\infty}^{2}}{\mu_{E}^{2}-\mu_{\infty}^{2}}=\frac{1}{2}$ ). Therefore, for dynamical QCD medium (in asymptotic limit), two thirds of the energy loss contribution comes from the longitudinal gluon exchange (electric contribution), while one third of the contribution comes from the transverse gluon exchange (magnetic contribution).

It was previously shown [9, 10] that, at asymptotically large jet energies, static and dynamical energy losses become equal up to a multiplicative constant $\frac{\lambda_{\mathrm{dyn}}}{\lambda_{\mathrm{stat}}}$ that can be renormalized. One should note that, in static QCD medium, only longitudinally polarized gluons give rise to the energy loss. Therefore, we have shown here that, while at asymptotically high energies, dynamical and static energy losses become numerically equal, their physical origin is different: For dynamical medium both longitudinally and transversely polarized gluons contribute to the energy loss, while for static medium there is only contribution from longitudinally polarized gluons.

\section{NUMERICAL ANALYSIS}

In this section we first use Eqs. (2.6) and (2.7) to study the importance of magnetic and electric contributions to the energy loss in dynamical QCD medium. The analysis is done in the energy range relevant for both RHIC and LHC experiments, and for the case of light, charm and bottom quarks.

In the numerical analysis we use the following parameters: We consider a quark-gluon plasma of temperature $T=225 \mathrm{MeV}$, with $n_{f}=2.5$ effective light quark flavors, and strong interaction strength $\alpha_{s}=0.3$, as representative of average conditions encountered in $\mathrm{Au}+\mathrm{Au}$ collisions at RHIC. For the light quark jets we assume that their mass is dominated by the thermal mass $M=\mu_{E} / \sqrt{6}$, where $\mu_{E}=g T \sqrt{1+N_{f} / 6} \approx 0.5 \mathrm{GeV}$ is the Debye screening mass. The charm mass is taken to be $M=1.2 \mathrm{GeV}$, and for the bottom mass we use $M=4.75 \mathrm{GeV}$. To simulate (average) conditions in $\mathrm{Pb}+\mathrm{Pb}$ collisions at the $\mathrm{LHC}$, we use the temperature of the medium of $T=400 \mathrm{MeV}$.

In Figs. 1 1 and 2 we compare the momentum dependence of the magnetic and electric contributions to the radiative energy loss in dynamical QCD medium. Figure 1 corresponds to RHIC conditions, while Fig. 2 corresponds to LHC conditions. The figures show that for all three types of quarks, and for both RHIC and LHC conditions, $i$ ) both electric and magnetic contributions play a significant part in the energy loss, ii) electric contribution is similar (though somewhat smaller) compared to the total energy loss in static QCD medium (note that in static QCD medium, only 

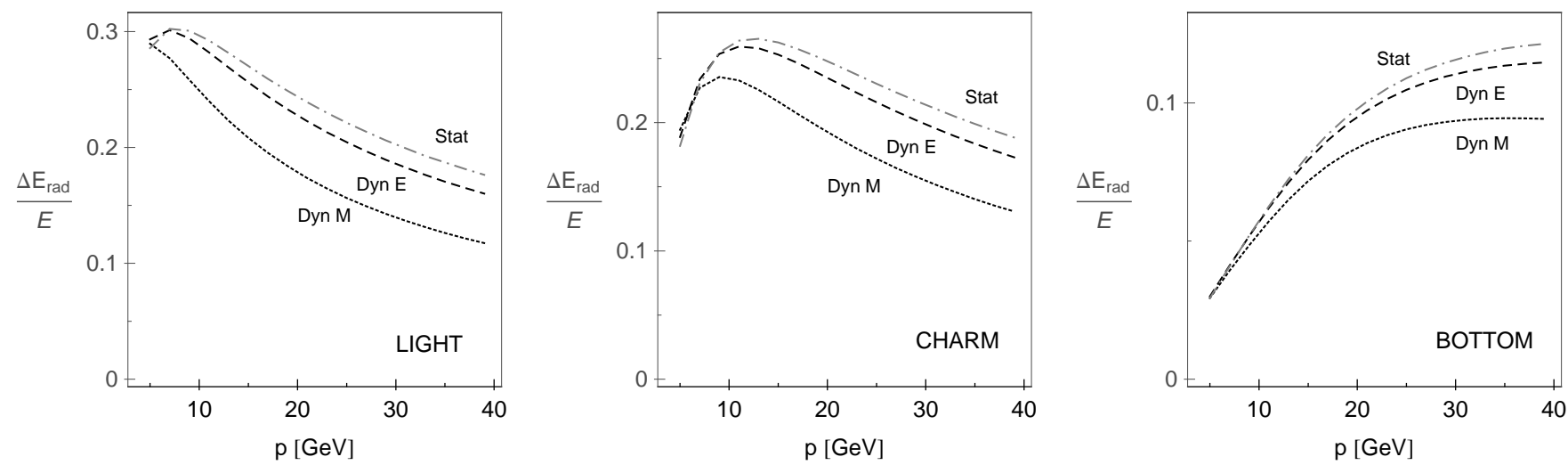

FIG. 1: Fractional radiative energy loss as a function of momentum for an assumed path length $L=5 \mathrm{fm}$ and a medium of temperature $T=225 \mathrm{MeV}$ ("RHIC conditions"). Left, center and right panels correspond respectively to light, charm and bottom quarks. Dotted and dashed curves correspond, respectively, to magnetic and electric contributions to the energy loss in a dynamical QCD medium, while dot-dashed curve corresponds to the energy loss in a static QCD medium (see [18]).
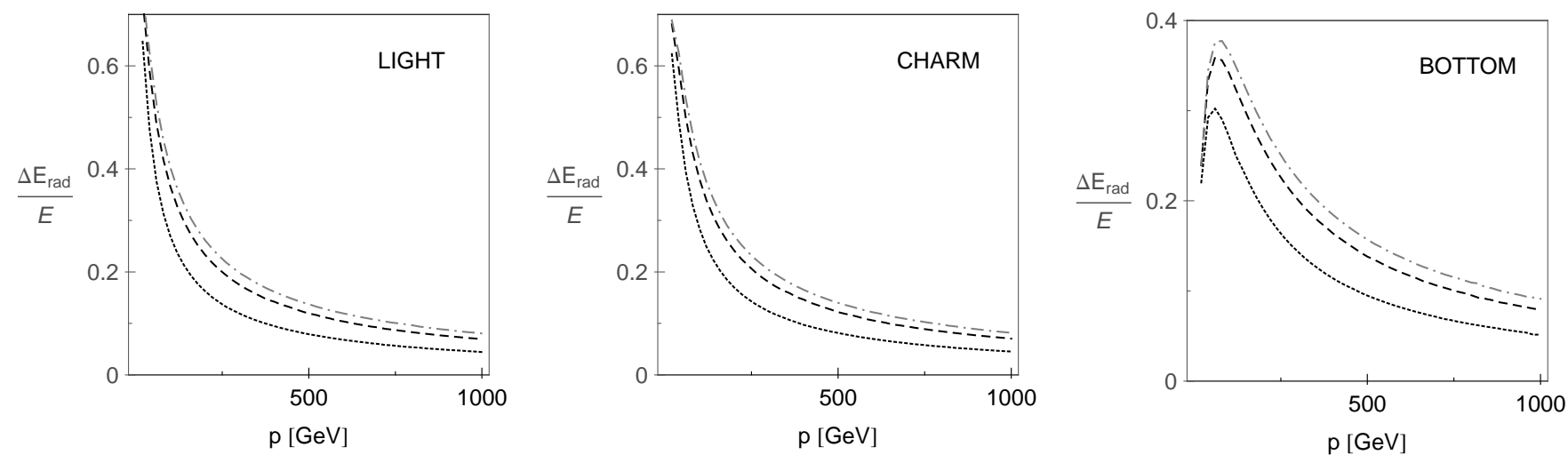

FIG. 2: Fractional radiative energy loss as a function of momentum for an assumed path length $L=5 \mathrm{fm}$ and a medium of temperature $T=400 \mathrm{MeV}$ ("LHC conditions"). Left, center and right panels correspond respectively to light, charm and bottom quarks. Dotted and dashed curves correspond, respectively, to magnetic and electric contributions to the energy loss in a dynamical QCD medium, while dot-dashed curve corresponds to the energy loss in a static QCD medium (see [18]).

electric contribution to the energy loss exists [12]). This leads to the conclusion that the increase in the energy loss in dynamical (relative to the static) QCD medium, exclusively comes from the magnetic contribution.

In Figure 3 we plot the transverse momentum dependence of the exchanged (the left panel) and emitted (the right panel) gluon spectrum. For both exchanged and emitted gluon spectrum, we observe similar behavior of the electric contribution in dynamical and static QCD medium; this is consistent with the similarity between electric contribution to the energy loss in dynamic and static QCD medium shown in Figs. 1 and 2 . On the other hand, we observe a qualitatively different momentum dependence for electric and magnetic contributions in the exchanged momentum spectrum (the left panel in Fig. 3). In particular, we see that magnetic contribution to the energy loss comes almost exclusively from low momentum spectrum (where $|\boldsymbol{q}| \lesssim 1 \mathrm{GeV}$ ). This difference between the electric and magnetic contributions does not appear in the emitted gluon spectrum (see the right panel in Fig. 31), which is a consequence of the fact that the difference between electric and magnetic contributions comes exclusively from $v_{T, L}(\boldsymbol{q})$ (that does not depend on $\boldsymbol{k}$ and is a property of the exchanged gluon only).

In Fig. 4 we analyze relative importance of magnetic and electric contributions for increasingly large values of jet energy. In order to see how the asymptotic limit discussed in the previous subsection is approached for energies at RHIC and LHC. Right (left) panel of the figure corresponds to the RHIC (LHC) case. For both cases, we see that relative importance of magnetic contribution decreases with the increase in jet energy, in similar way for all three types of quarks. We note that, for asymptotically large jet energies, the ratio will reach a limiting value of $1 / 2$, consistently with the analytical result shown in the previous section (data not shown). 

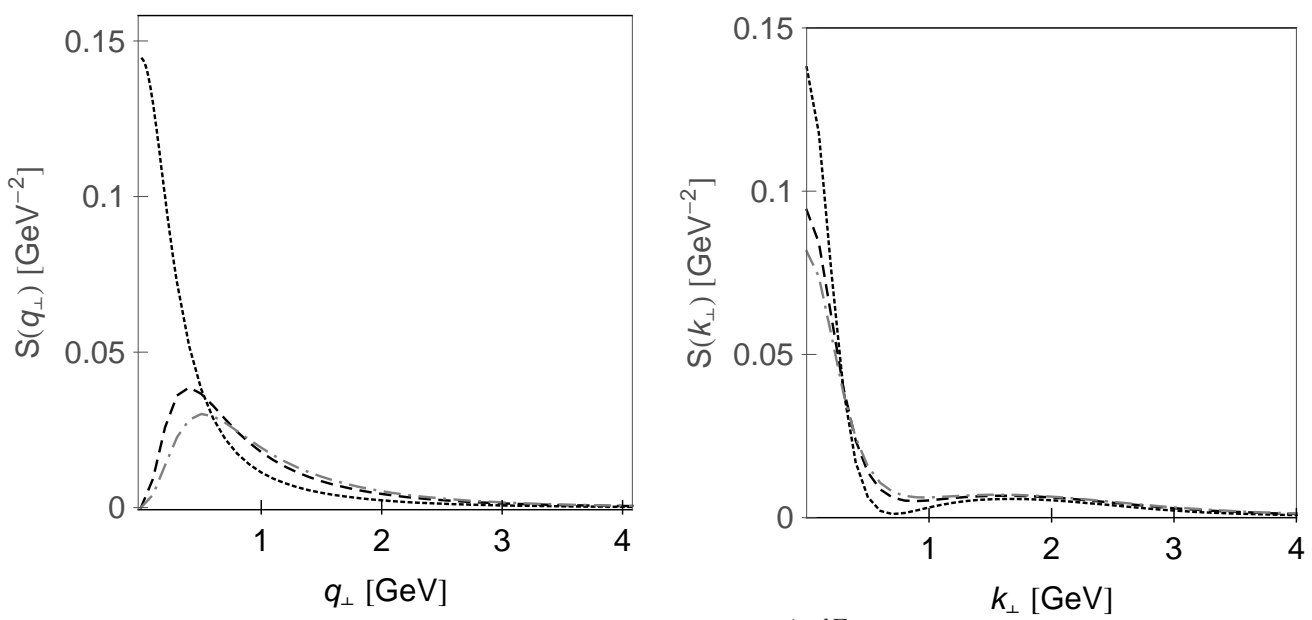

FIG. 3: Transverse momentum spectrum of the exchanged $\left(S(\boldsymbol{q}) \equiv \frac{1}{E} \frac{d E}{d^{2} \boldsymbol{q}}\right.$, left panel) and emitted $\left(S(\boldsymbol{k}) \equiv \frac{1}{E} \frac{d E}{d^{2} \boldsymbol{k}}\right.$, right panel) gluons for light quarks traveling for $L=5 \mathrm{fm}$ through a dynamical or static QCD medium. Assumed temperature of the medium is $T=225 \mathrm{MeV}$ ("RHIC conditions"). Dotted and dashed curves correspond, respectively, to magnetic and electric contributions to the transverse momentum spectrum in a dynamical QCD medium, while dot-dashed curve corresponds to the momentum spectrum in a static QCD medium. The initial charm quark energy is assumed to be $20 \mathrm{GeV}$. Note that $|\boldsymbol{q}|$ and $|\boldsymbol{k}|$ are denoted as $\mathrm{q}_{\perp}$ and $\mathrm{k}_{\perp}$ in the figure.
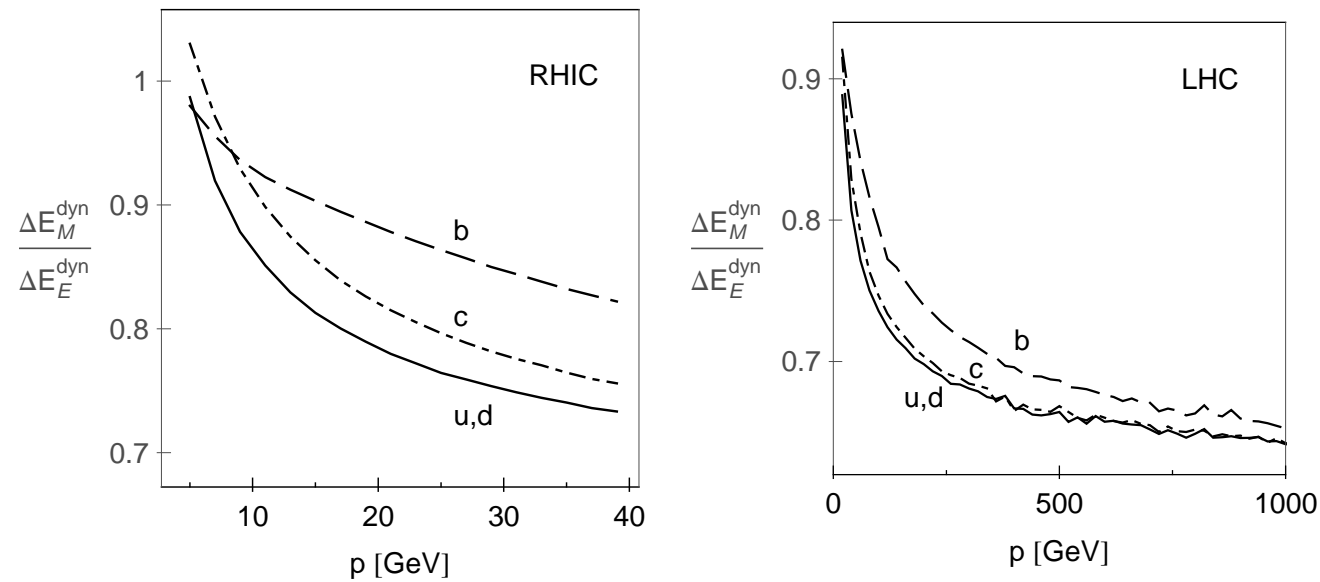

FIG. 4: Ratio of magnetic and electric contributions to the radiative energy loss in a finite size dynamical QCD medium for light, charm and bottom quark (full, dot-dashed and dashed curve, respectively). Left (right) panel corresponds to the RHIC (LHC) case, with assumed temperature $T=225 \mathrm{MeV}(T=400 \mathrm{MeV})$.

\section{SUMMARY}

In this paper we studied the origin of the energy loss increase in dynamical QCD medium relative to static QCD medium. While energy loss in static QCD medium has only electric contribution from the gluon exchange, we find that, in dynamical QCD medium, both electric and magnetic contributions exist and are comparable. Since electric contributions in static and dynamical QCD medium are approximately equal at RHIC and LHC energies, the boost of energy loss results relative to the static QCD medium comes entirely from the additional magnetic contribution in the dynamical QCD medium. Furthermore, while energy loss in dynamical QCD medium approaches the static results for asymptotically high energies, the physical origin of the energy loss is different for dynamical and static case in this limit: In the static case, the entire energy loss comes from the electric contribution, while for dynamical medium one third of the energy loss comes from magnetic contribution.

The calculation presented here is based on HTL perturbative QCD, which requires zero magnetic mass. On the other hand, different non-perturbative approaches [19 22] suggest a non-zero magnetic mass for RHIC and LHC case. Since this paper established significance of the magnetic contribution in the radiative energy loss, the work presented here therefore opens the following question: Is it possible to consistently include finite magnetic mass in the dynamical energy loss calculations, and how this inclusion would modify energy loss result? Addressing this question is our next important goal. 


\section{Acknowledgments}

This work is supported by Marie Curie International Reintegration Grant within the $7^{\text {th }}$ European Community Framework Programme (PIRG08-GA-2010-276913) and by the Ministry of Science and Technological Development of the Republic of Serbia, under projects No. ON171004 and ON173052.

[1] J.D. Bjorken: FERMILAB-PUB-82V059-THY (1982) 287, 292

[2] N. Brambilla et al., Preprint hep-ph/0412158 (2004).

[3] M. Gyulassy, Lect. Notes Phys. 583, 37 (2002).

[4] D. d'Enterria and B. Betz, Lect. Notes Phys. 785, 285 (2010).

[5] M. Guylassy, I. Vitev, X. N. Wang and B. W. Zhang, in Quark Gluon Plasma 3, edited by R. C. Hwa and X. N. Wang, p. 123 (World Scientific, Singapore, 2003)

[6] R. Baier, Yu. L. Dokshitzer, A. J. Mueller and D. Schiff, Phys. Rev. C 58, 1706 (1998).

[7] R. Baier, D. Schiff, B. G. Zakharov, Ann. Rev. Nucl. Part. Sci. 50, 37 (2000).

[8] A. Kovner and U. A. Wiedemann, in Quark Gluon Plasma 3, edited by R.C. Hwa and X.N. Wang, p. 192 (World Scientific, Singapore, 2003) arXiv:hep-ph/0304151.

[9] M. Djordjevic, Phys. Rev. C 80, 064909 (2009).

[10] M. Djordjevic and U. Heinz, Phys. Rev. Lett. 101, 022302 (2008).

[11] M. Gyulassy, Physics 2, 107 (2009).

[12] M. Djordjevic and U. Heinz, Phys. Rev. C 77, 024905 (2008).

[13] P. Aurenche, F. Gelis and H. Zaraket, JHEP 0205, 043 (2002).

[14] U. Heinz, Ann. Phys. (N.Y.) 168, 148 (1986).

[15] R.D. Pisarski, Physica A 158, 146 (1989).

[16] M. Le Bellac, Thermal Field Theory (Cambridge University Press, 1996).

[17] A. Adil, M. Gyulassy, W. A. Horowitz and S. Wicks, Phys. Rev. C 75, 044906 (2007).

[18] M. Djordjevic and M. Gyulassy, Phys. Lett. B 560, 37 (2003); and Nucl. Phys. A 733, 265 (2004).

[19] Yu. Maezawa et al. [WHOT-QCD Collaboration], arXiv:1003.1361 [hep-lat]; Yu. Maezawa et al. [WHOT-QCD Collaboration], PoS Lattice 194 (2008).

[20] A. Nakamura, T. Saito and S. Sakai, Phys. Rev. D 69, 014506 (2004).

[21] A. Hart, M. Laine and O. Philipsen, Nucl. Phys. B 586, 443 (2000).

[22] D. Bak, A. Karch and L. G. Yaffe, JHEP 0708, 049 (2007). 